\title{
Modification of splenic stiffness on acoustic radiation force impulse parallels the variation of portal pressure induced by transjugular intrahepatic portosystemic shunt
}

\author{
Adriano De Santis, ${ }^{*}$ Silvia Nardelli, ${ }^{*}$ Chiara Bassanelli, ${ }^{*}$ Marinella Lupo, ${ }^{*}$ Claudia legri, * \\ Carmela Anna Di Ciesco, ${ }^{*}$ Mariana Forlino, ${ }^{*}$ Alessio Farcomeni ${ }^{\dagger}$ and Oliviero Riggio*
}

Departments of * Clinical Medicine, Gastroenterology Unit and Center for the study of Portal Hypertension, and ${ }^{\dagger}$ Public Health and Infectious Diseases, Sapienza University of Rome, Rome, Italy

\section{Key words}

ARFI imaging, liver stiffness, portal

hypertension, spleen stiffness, TIPS.

Accepted for publication 17 July 2017.

\section{Correspondence}

Dr Silvia Nardelli, Department of Clinical

Medicine, Gastroenterology Unit and Center for the study of Portal Hypertension, Sapienza University of Rome, Piazzale Aldo Moro, 5, 00185 Rome, Italy.

Email: nardelli.silvia@gmail.com

Declaration of conflict of interest: The authors declare that no conflict of interest exists concerning this paper.

Author contributions: Adriano De Santis works on the study concept and design; acquisition, analysis, and interpretation of data; and study supervision; Silvia Nardelli and Chiara Bassanelli were responsible for the acquisition and analysis of data, collection of the data, statistical analysis, and manuscript preparation; Marinella Lupo, Claudia legri, Carmela Anna Di Ciesco, and Mariana Forlino also took part in the acquisition of data; Alessio Farcomeni contributed in the statistical analysis of data; and Oliviero Riggio also worked on the study concept and design; analysis and interpretation of data; manuscript preparation; final drafting of the manuscript; and study supervision.

\begin{abstract}
Background and Aim: Spleen and liver stiffness (LS) measured by acoustic radiation force impulse (ARFI) imaging has been shown to be useful in identifying patients with portal hypertension. The study aims to establish if the modification of portal pressure induced by a transjugular intrahepatic portosystemic shunt (TIPS) parallels the modification of spleen or LS measured by ARFI in order to understand if ARFI may be used to monitor the modification of portal pressure in patients with cirrhosis.

Methods: Thirty-eight patients with severe portal hypertension underwent LS and spleen stiffness (SS) before TIPS and 1 week after TIPS. Portal atrial gradient (PAG) was measured before and after the shunt opening.

Results: Portal atrial gradient decreased significantly from 19.5 to $6 \mathrm{mmHg}(P<0.001)$. SS decreased significantly after TIPS (pre-TIPS $3.7 \mathrm{~m} / \mathrm{s}$ vs post-TIPS $3.1 \mathrm{~m} / \mathrm{s}$; $P<0.001$ ), and LS was also significantly modified by TIPS (pre-TIPS $2.8 \mathrm{~m} / \mathrm{s} v s$ postTIPS $2.4 \mathrm{~m} / \mathrm{s} ; P=0.003)$. PAG and SS values measured before and after TIPS were significantly correlated $(r=0.56 ; P<0.001)$; on the other hand, PAG and LS were not $(r=0.19$; $P=0.27$ ). Two patients developed a persistent hepatic encephalopathy refractory to medical treatment and were submitted to the reduction of the stent diameter. The modification of SS was parallel to the modification of PAG.

Conclusion: Spleen stiffness is superior to LS in detecting the modification of portal pressure induced by TIPS. This makes SS a potential non-invasive method to monitor the modification of portal hypertension. Further investigations are needed to establish applicability and clinical utility of this promising tool in the treatment of portal hypertension.
\end{abstract}

\section{Introduction}

Acoustic radiation force impulse (ARFI) imaging ${ }^{1}$ is an ultrasound-based technique in which shear wave velocity is evaluated to assess the elastic properties of target tissues. ARFI has been proposed as an alternative method to assess tissue elasticity., ${ }^{2,3}$ ARFI can be performed under clear observation of the actual measuring site by B-mode imaging ${ }^{4}$ and thus may be more feasible than tissue elastometry, which may be difficult in patients with ascites, obesity, or narrow intercostal space. Promising results on the accuracy of ARFI technology for non-invasive assessment of liver fibrosis have been reported.,
Splenomegaly in liver cirrhosis is believed to be a consequence of portal congestion and tissue hyperplasia and to be strongly related to the degree of portal hypertension. ${ }^{5-7}$ Spleen diameter and platelets count have been related to the severity of portal hypertension and included in several models to predict the presence of clinically significant portal hypertension or esophageal varices. ${ }^{8,9}$ Spleen stiffness (SS) measured by tissue elastometry has been showed to be significantly correlated to the hepatic venous pressure gradient. ${ }^{10}$ Moreover, the capacity of SS was measured either by tissue elastometry ${ }^{10-12}$ or by ARFI ${ }^{13}$ in identifying patients with portal hypertension (i.e., hepatic venous 
pressure gradient $[\mathrm{HVPG}]>10 \mathrm{mmHg}$ ); clinically significant portal hypertension (HVPG $>12 \mathrm{mmHg}$ ) or varices at endoscopy has been shown to be superior to liver stiffness (LS) although not in all studies. ${ }^{14-16}$ Thus, SS can be considered a promising method for the non-invasive diagnosis of portal hypertension. ${ }^{17-19}$ However, little is known on the relationship between the modification of the portal pressure and the modification of splenic stiffness. The demonstration of the possibility that changes in portal pressure could be estimated accurately by changes in SS would have relevant clinical consequence, especially for the treatment of portal hypertension.

Transjugular intrahepatic portosystemic shunt (TIPS) is a radiological technique used for the treatment of the complications of portal hypertension, especially in patients with recurrent variceal bleeding or refractory ascites. ${ }^{20}$ TIPS construction leads to an immediate and large modification of portal pressure, which in most cases reaches normal values immediately after the shunt opening.

The aim of the present study was to establish if the modification of portal pressure induced by TIPS parallels the modification of spleen or LS measured by ARFI; in order to understand whether or not ARFI may contribute to indirect monitoring, the modification of portal pressure in patients with portal hypertension.

\section{Methods}

Between September 2013 and December 2015, all 48 consecutive patients with portal hypertension submitted to TIPS were considered for this prospective study. Ten patients submitted to rescue TIPS were not included because of the impossibility in performing the ARFI examinations before the procedure for practical problems during the emergency procedure.

The 38 patients included in the study had a clinically significant portal hypertension complicated by variceal bleeding or ascites refractory to conventional non-derivative treatment. All patients were affected by liver cirrhosis. However, one patient was affected by concomitant schistosomiasis and HCV infection, while two patients were affected by a Budd Chiari syndrome. In all these three patients, the cirrhosis was biopsy proven. The characteristics of the patients are reported in Table 1.

All TIPS procedures were carried out by the same radiologist. PTFE-covered stent-grafts (Gore Viatorr TIPS endoprosthesis, W.L. Gore \& Associates, Inc., Flagstaff, AZ, USA) were used. The anesthesiological procedure ${ }^{21-23}$ and the technical details of TIPS implantation with PTFE-covered stent grafts were previously described. ${ }^{24,25}$ All subjects were evaluated and followed up by the same medical team by a prospective protocolized diagnostic workup and a surveillance strategy as previously described. ${ }^{24,25}$ Before TIPS, the patients' histories were collected; physical examinations, calculations of Child-Pugh's ${ }^{26}$ and MELD ${ }^{27}$ scores, and Doppler ultrasonography and upper gastrointestinal endoscopy were carried out.

An informed, written consent was obtained. The Sapienza University of Rome Ethical Committee approved the study (Rif.1720/ 01.10.09).

Portal atrial gradient determination. The portal atrial gradient (PAG) was calculated as the difference between the portal
Table 1 Demographic and clinical characteristics of the patients evaluated for the study

\begin{tabular}{ll}
\hline & Pts enrolled in the study ${ }^{38}$ \\
\hline Age (years) & $55(47.5,63)$ \\
Gender (male/female) & $28 / 10$ \\
Etiology of liver disease ( $n)$ & \\
Virus & 13 \\
Alcohol & 15 \\
Budd-Chiari syndrome & 2 \\
Autoimmune & 2 \\
NASH & 3 \\
Cryptogenic & 2 \\
Schistosomiasis & 1 \\
Child-Pugh score & $8(7,9)$ \\
Child-Pugh class A/B/C ( $n)$ & $8 / 28 / 2$ \\
MELD score & $11(9,15)$ \\
INR & $1.37(1.25,1.60)$ \\
Bilirubin (mg/dl) & $1.21(0.94,2.55)$ \\
Albumin (g/dl) & $3.35(3,3.6)$ \\
Creatinine (mg/dl) & $0.9(0.7,1)$ \\
TIPS indication ( $n$ ) & 17 \\
$\quad$ Variceal bleeding & 21 \\
Refractory ascites &
\end{tabular}

Results expressed as median (first quartile and third quartile) or counts. INR, international normalized ratio; MELD, model of end-stage liver disease; NASH, non-alcoholic steatohepatitis; TIPS, transjugular intrahepatic portosystemic shunt.

and the atrial pressure. ${ }^{28}$ PAG was measured in the deeply sedated (not intubated) patient by means of a pressure transducer before and immediately after TIPS construction. The first PAG measurement was obtained after the portal puncture, once the catheter is advanced into a patent portion of the main portal vein. The second PAG measurement was obtained after the deployment of the covered stent between the portal and hepatic vein, balloon dilatation of the stent with a balloon catheter of size equivalent to the nominal diameter of the graft and after a portography showing the shunt patency.

A recent Spanish study ${ }^{29}$ has proved that the portosystemic gradient measured a few days after TIPS opening is more accurate than the one measured immediately after TIPS. Although the difference between the two measurements was in the order of $1.5 \mathrm{mmHg}$.

Ultrasound and Doppler examination. A complete ultrasound and Doppler examination was performed in each patient before TIPS by three expert operators (ADS with more than 30 years of experience and $\mathrm{CB}$ and $\mathrm{CI}$ with almost 10 years of experience). Each exam was conducted to record the spleen bipolar and the liver right lobe diameters, portal, splenic, and mesenteric vein diameter and the portal flow velocity and direction. One week after TIPS, ultrasonography assessment of the shunt patencywith color-Doppler measurement of the mean flow velocity and direction within the shunt at three sites (proximal, medial, and distal portion), in the portal vein and in the intrahepatic portal branches was obtained. The diameters of the stent, the portal vein, and the spleen were also measured. 
Spleen and liver stiffness determination. All ARFI measurements were obtained using a Siemens Acuson S2000 ultrasound system by the same experienced sonographer (ADS). After an overnight fast, each patient was placed in the supine position and underwent ARFI on B-mode imaging during deep inspiration. ${ }^{30} \mathrm{~A}$ region of interest (fixed-dimension $1 \times 0.5-\mathrm{cm}$ box; maximum evaluable depth, $8.5 \mathrm{~cm}$ ) in the liver or spleen parenchyma, free of large blood vessels, was selected using the intercostal approach. Liver stiffness was measured in the right lobe of the liver, almost $1 \mathrm{~cm}$ below the liver capsule, using the intercostal approach. Ten consecutive successful measurements were performed in each patient, and mean value in meters per second was considered as results.

In the first three patients, spleen and LS were measured the day before TIPS and $24 \mathrm{~h}$ and 7 days after TIPS. Values of SS measured $24 \mathrm{~h}$ after TIPS were only partially decreased in comparison with those obtained 1 week after TIPS (from $3.64 \pm 0.43$ to $3.05+0.17$ and to $2.66+0.22 \mathrm{~m} / \mathrm{s} ; P<0.001)$. Therefore, in the remaining patients, ARFI was performed only a week after the stent placement.

Statistical analysis. Results were expressed as median (first quartile and third quartile). Comparisons between groups were performed for quantitative data by Wilcoxon's paired rank-sum test paired data and chi-squared test when appropriate. The relationship between variables was analyzed by Spearman's correlations and related test. The software $\mathrm{R}$ ( $\mathrm{R}$ Development Core Team, Vienna, Austria) was used for the computations.

\section{Results}

Transjugular intrahepatic portosystemic shunt was successfully implanted in all patients. The PAG measured immediately before and after TIPS as well as the ultrasound/Doppler parameters and the spleen and LS values measured before and 1 week after TIPS are reported in Table 2. At the time of the second measurement, the shunts were patent in all patients.

Immediately after stent-graft placement, the portal pressure gradient decreased significantly from a median of 19.5 to $6 \mathrm{mmHg}$ $(P<0.001)$. SS decreased significantly after TIPS (pre-TIPS
$3.7 \mathrm{~m} / \mathrm{s} v s$ post-TIPS $3.1 \mathrm{~m} / \mathrm{s} ; P<0.001$ ), and also, LS was significantly modified by TIPS (pre-TIPS $2.8 \mathrm{~m} / \mathrm{s} v s$ post-TIPS $2.4 \mathrm{~m} / \mathrm{s} ; P=0.003)$.

One week after TIPS, all ultrasound parameters remained stable compared with basal values, whereas portal flow velocity was significantly increased from a median of 12.7 to $21 \mathrm{~cm} / \mathrm{s}$ $(P<0.001$ at Wilcoxon test). SS and LS decreased significantly (Table 2).

Figure 1 reports the individual values of portal pressure, SS, and LS recorded before and after TIPS in each patient. One week after TIPS, splenic stiffness was reduced in 35 out of 38 patients. In the remaining three patients, SS remained stable in two and was slightly increased of about $10 \%$ in one patient. In this patient, the measurements were repeated 1 month after the procedure and the value resulted lower than those recorded before and 1 week after TIPS (3.23 before TIPS, 3.371 week after TIPS and $2.58 \mathrm{~m} / \mathrm{s} 1$ month after TIPS). It was not possible to repeat the ARFI measurements in the remaining two patients because one patient died 1 week after discharge for liver failure due to acute alcoholic hepatitis and the other patients was detained. At variance with splenic stiffness, the modification of LS after TIPS was highly variable (Figure 1). The larger reduction was observed in the two patients with Budd Chiari syndrome.

Using Spearman's test, only PAG and SS values measured before and after TIPS were significantly correlated (as shown in Figure 2: $r=0.56 ; P<0.001)$, while PAG and LS were not $(r=0.19 ; P=0.27)$.

Two patients developed a persistent hepatic encephalopathy refractory to medical treatment 1 and 8 months after TIPS placement, respectively, and were submitted to the reduction of the stent diameter, as previously described. ${ }^{21,22}$ In these patients, PAG gradient was determined before and after the reduction of the stent diameter, while liver and SS were performed the day before and 1 week after the radiological procedure. In one of these two patients, ascites and large esophageal varices were again evident 1 month after the stent diameter reduction and the patient was submitted to a re-dilatation of the stent. Figure 3 reports the modifications of PAG and SS observed in these two patients before, after TIPS, and after the stent diameter modifications. A parallel modification of SS and PAG was observed. No significant variation of LS was observed in these patients.

Table 2 Portal pressure gradient, ultrasound and Doppler parameters, and spleen and liver stiffness values measured before and after TIPS

\begin{tabular}{|c|c|c|c|}
\hline & Before TIPS $(n=38)$ & After TIPS $(n=38)$ & $P$ \\
\hline Portal pressure gradient $(\mathrm{mmHg})^{*}$ & $19.5(16,21.5)$ & $6(5,8.25)$ & $<0.001$ \\
\hline Splenic stiffness (m/s) & $3.7(3.42,3.99)$ & $3.1(2.72,3.37)$ & $<0.001$ \\
\hline Liver stiffness (m/s) & $2.8(2.5,3.1)$ & $2.4(2.2,2.6)$ & 0.003 \\
\hline Spleen longitudinal diameter (cm) & $16.4(14.9,19.5)$ & $16.3(14.3,18.5)$ & 0.09 \\
\hline Liver diameter (cm) & $14.2(12.7,15.7)$ & $13.8(11.9,15.7)$ & 0.09 \\
\hline Splenic vein diameter $(\mathrm{cm})(n=21)$ & $10.9(9.7,13.3)$ & $10.9(9.4,12.2)$ & 0.22 \\
\hline Portal vein diameter (mm) & $14(13.1,15.7)$ & $13.9(12.4,15.4)$ & 0.13 \\
\hline Mesenteric vein diameter $(\mathrm{mm})$ & $11.3(9.9,12.9)$ & $11(9.4,12.1)$ & 0.09 \\
\hline Portal flow mean velocity $(\mathrm{cm} / \mathrm{s})(n=17)$ & $12.7(10.7,13.8)$ & $25.1(19.6,34.5)$ & $<0.001$ \\
\hline
\end{tabular}

${ }^{*}$ After TIPS, PPG was measured immediately after the stent implantation and deployment. All the other parameters were measured before and 1 week after TIPS.

Results are expressed as median (first quartile and third quartile); Wilcoxon test was used.

PPG, portal pressure gradient; TIPS, transjugular intrahepatic portosystemic shunt. 


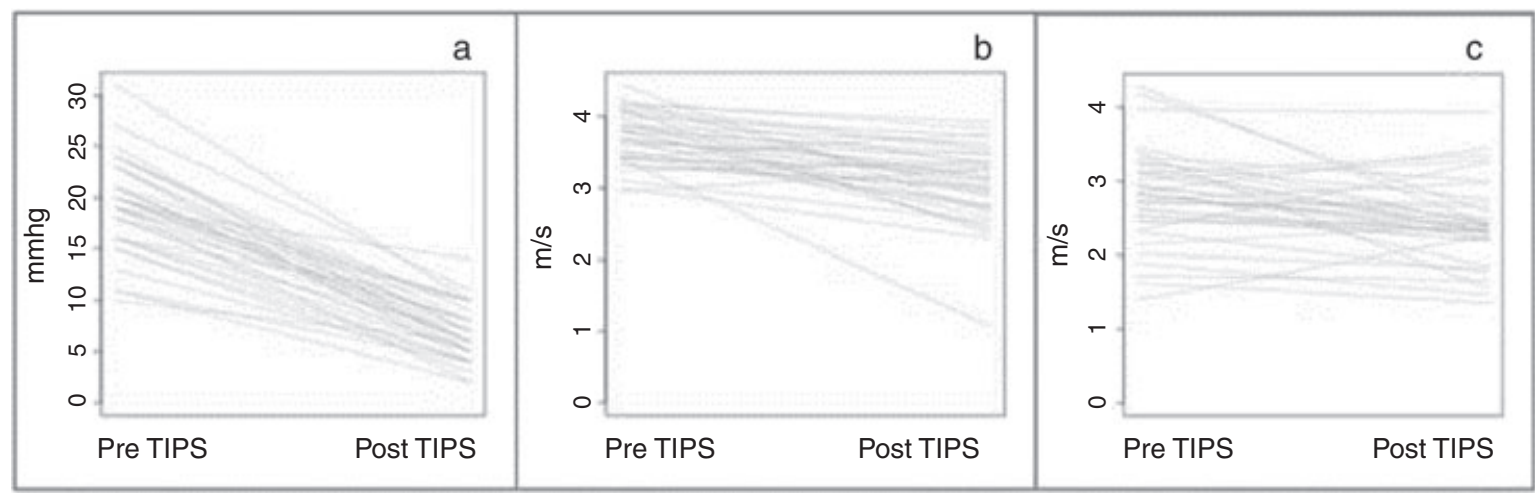

Figure 1 Individual values of portal pressure (panel a) measured immediately before and after transjugular intrahepatic portosystemic shunt (TIPS) and of spleen stiffness (panel b) and liver stiffness (panel c) measured before and 1 week after TIPS. Wilcoxon's rank-sum test was used for statistical analysis.

\section{Discussion}

Non-invasive assessment of portal hypertension has become an issue of growing importance over the past years. Although the portosystemic pressure gradient is considered the standard of care for patients with liver cirrhosis and portal hypertension, alternative non-invasive surrogate indicators are being evaluated for the presence of complications of portal hypertension and the efficacy of TIPS treatment. Given the high frequency of asymptomatic shunt failure, an accurate screening test is needed to confirm shunt effectiveness and to identify shunt malfunctions. Alternative noninvasive methods would minimize both patient morbidity and costs. $^{31-33}$

In this study, we showed that the reduction of portal pressure gradient induced by TIPS led to a significant reduction of the SS and LS measured by ARFI imaging; but using correlation test, only SS was significantly correlated to PAG decrease after TIPS. According to our results, the change of the SS after

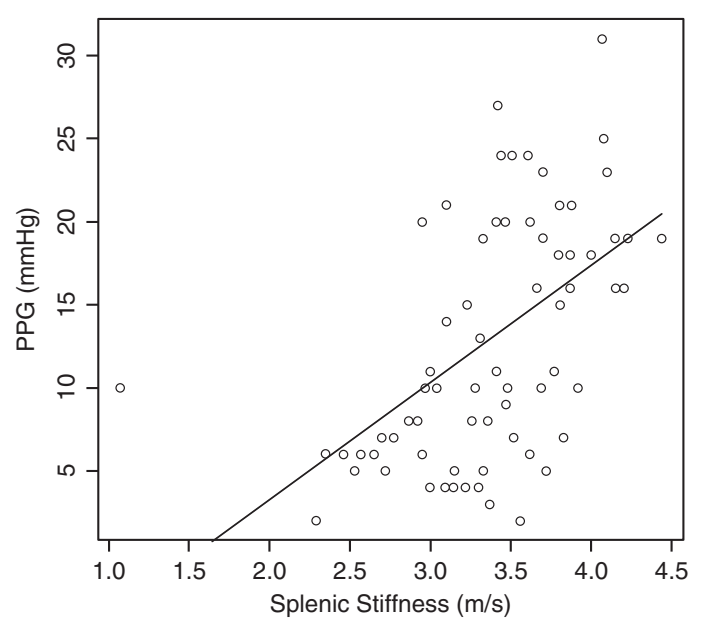

Figure 2 Correlation between portal pressure gradient (PPG) and spleen stiffness values measured before and after transjugular intrahepatic portosystemic shunt (Spearman's correlation: $r=0.56$ $P<0.001)$.
TIPS placement may be useful as a predictive marker of the status of the TIPS.

Interestingly, in the two patients with persistent post-TIPS hepatic encephalopathy in whom the stent diameter was modified, the SS paralleled the portal pressure gradient modifications induced by the reduction or re-dilatation of the stent. SS, although related also to hepatic fibrosis, ${ }^{34}$ seems to be more sensitive to the variation of portal pressure probably because the splenic pressure is more directly dependent on the splenic vein outflow obstruction and on the hyperdynamic circulation due to the exclusive arterial supply of the spleen as suggested by the significantly correlated, in our series, PAG and SS values measured before and after TIPS were. It should be noted that PAG and SS were measured in different times: immediately after the shunt opening and after 1 week, respectively. Preliminary measurements performed in few patients showed that SS may continue to decrease 1 week after a TIPS compared with the values observed $24 \mathrm{~h}$ later. This may also occur for PAG measurements due to adaptation of the hearth to the opening of the shunt. Thus, the correlation between PAG and SS observed in our experimental condition could be much better if the two measurements could be made at the same time.

The parallelism between SS and portal pressure confirms the preliminary observations of $\operatorname{Ran}^{31}$ and $\mathrm{Gao}^{32}$ in 12 and 10 patients, respectively, and is at variance with the study of Novelli et $a l .^{33}$ in which the correlation between the variation of SS and portal pressure was not observed in a cohort of 19 patients.

The increase in SS after the reduction of the stent diameter observed in the two patients with persistent hepatic encephalopathy suggests that in patients submitted to TIPS, this parameter may be used to monitor the shunt function, confirming the observation of Gao et al. ${ }^{35}$ However, the efficacy and the sensitivity of splenic stiffness in detecting the occurrence of a shunt dysfunction after TIPS needs further investigations.

The use of splenic stiffness measurement as a non-invasive method to detect the presence of clinically significant portal hypertension ${ }^{10}$ or of esophageal varices ${ }^{13}$ was already suggested, although the models based on SS for the identification of the patients with large varices need to be fully validated. ${ }^{36,37}$ SS has been showed to be useful also in patients with portal hypertension due to portal vein obstruction. ${ }^{18}$ In addition to the above 


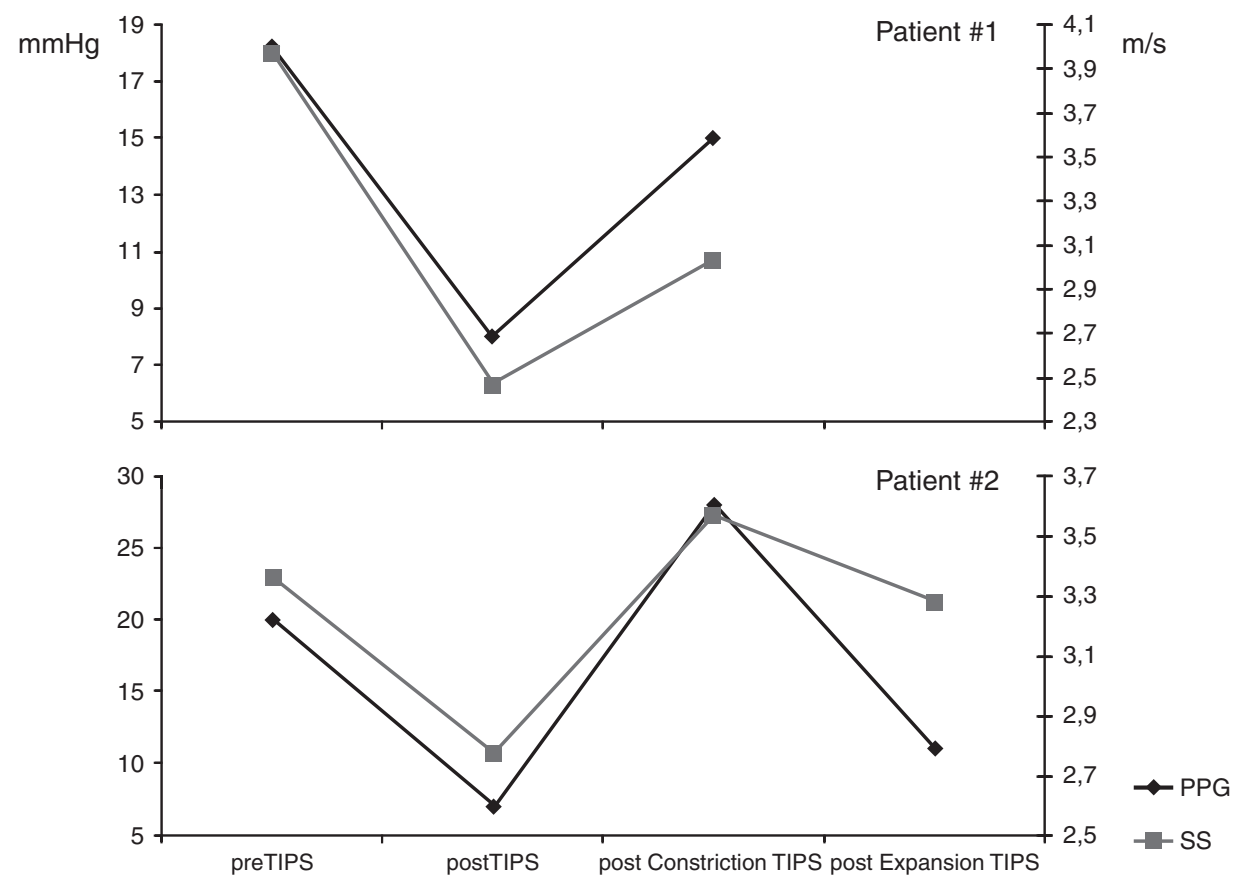

Figure 3 Modification of portal pressure gradient ( $\rightarrow-$, PPG: black line) and spleen stiffness (---, SS: grey line) in the two patients who needed to be submitted to modification of the shunt diameter because of refractory hepatic encephalopathy.

applications, our findings support the possibility that the modification of portal pressure could be monitored by SS measurement by ARFI imaging. This possibility has clinical implications. Portal pressure has been showed to have a prognostic impact on survival and development of the complications in patients with liver cirrhosis. ${ }^{38,39}$ Moreover, at variance with arterial hypertension, the pharmacological treatment of portal hypertension is usually carried on without monitoring the portal pressure. Recent data suggest that less than half of the patients treated with beta blockers respond in terms of portal pressure reduction. ${ }^{40}$ Until now, HVPG is the only reliable method to estimate the degree of portal hypertension and its reduction during treatment. However, the diffusion and the applicability of HVPG measurements are limited by its invasiveness. Our findings, although promising, are far to let consider SS measurement as a reliable method to monitor little modifications of portal pressure, like those induced by a pharmacological therapy. Thus, studies with measurement of HVPG and SS before and after a pharmacological treatment are needed to understand if SS modifications are sufficiently sensitive to detect the druginduced modification of portal pressure.

In conclusion, SS, more than LS, is useful to detect the modification of portal pressure gradient induced by TIPS. This makes SS a potential non-invasive method to monitor the stent function and the modification of portal hypertension. Further investigations are needed to establish applicability and clinical utility of this promising tool.

\section{References}

1 Nightingale K, Bentley R, Trahey G. Observations of tissue response to acoustic radiation force: opportunities for imaging. Ultrason. Imaging 2002; 24: 129-38.
2 Friedrich-Rust M, Wunder K, Kriener S et al. Liver fibrosis in viral hepatitis: noninvasive assessment with acoustic radiation force impulse imaging versus transient elastography. Radiology 2009; 252: 595-604.

3 Takahashi H, Ono N, Eguchi Y et al. Evaluation of acoustic radiation force impulse elastography for fibrosis staging of chronic liver disease: a pilot study. Liver Int. 2010; 30: 538-45.

4 Friedrich-Rust M, Nierhoff J, Lupsor M et al. Performance of acoustic radiation force impulse imaging for the staging of liver fibrosis: a pooled meta-analysis. J. Viral Hepat. 2012; 19: 212-19.

5 Chalasani N, Imperiale TF, Ismail A et al. Predictors of large esophageal varices in patients with cirrhosis. Am. J. Gastroenterol. 1999; 94: 3285-91.

6 Madhotra R, Mulcahy HE, Willner I, Reuben A. Prediction of esophageal varices in patients with cirrhosis. J. Clin. Gastroenterol. 2002; 34: 81-85.

7 De Franchis R. Non invasive diagnosis of esophageal varices: is it feasible? Am. J. Gastroenterol. 2006; 101: 2520-2.

8 Giannini E, Botta F, Borro P et al. Platelet count/spleen diameter ratio: proposal and validation of a non-invasive parameter to predict the presence of oesophageal varices in patients with liver cirrhosis. Gut. 2003; 52: 1200-5.

9 Berzigotti A, Seijo S, Arena U et al. Elastography, spleen size, and platelet count identify portal hypertension in patients with compensated cirrhosis. Gastroenterology 2013; 144: 102-11.

10 Colecchia A, Montrone L, Scaioli E et al. Measurement of spleen stiffness to evaluate portal hypertension and the presence of esophageal varices in patients with HCV-related cirrhosis. Gastroenterology 2012; 143: 646-54.

11 Sharma P, Kirnake V, Tyagi V et al. Spleen stiffness in patients with cirrhosis in predicting esophageal varices. Am. J. Gastroenterol. 2013; 108: 1101-7.

12 Calvaruso V, Bronte F, Conte E, Simone F, Craxì A, Di Marco V. Modified spleen stiffness measurement by transient elastography is associated with presence of large oesophageal varices in patients with compensated hepatitis C virus cirrhosis. J. Viral Hepat. 2013; 20: 867-74.

13 Takuma Y, Nouso K, Morimoto Y et al. Measurement of spleen stiffness by acoustic radiation force impulse imaging identifies cirrhotic patients with esophageal varices. Gastroenterology 2013; 144: 92-101. 
14 Takuma Y, Nouso K, Morimoto Y et al. Portal hypertension in patients with liver cirrhosis: diagnostic accuracy of spleen stiffness. Radiology. 2016; 279: 609-19.

15 Zykus R, Jonaitis L, Petrenkienė V, Pranculis A, Kupčinskas L. Liver and spleen transient elastography predicts portal hypertension in patients with chronic liver disease: a prospective cohort study. BMC Gastroenterol. 2015; 24: 183.

16 Elkrief L, Rautou PE, Ronot M et al. Prospective comparison of spleen and liver stiffness by using shear-wave and transient elastography for detection of portal hypertension in cirrhosis. Radiology. 2015; 275: 589-98.

17 Furuichi Y, Moriyasu F, Taira J et al. Noninvasive diagnostic method for idiopathic portal hypertension based on measurements of liver and spleen stiffness by ARFI elastography. J. Gastroenterol. 2013; 48: 1061-8.

18 Sharma P, Mishra SR, Kumar M, Sharma BC, Sarini SK. Liver and spleen stiffness in patients with extrahepatic portal vein obstruction. Radiology 2012; 263: 893-9.

19 Jansen C, Bogs C, Verlinden W et al. Shear-wave elastography of the liver and spleen identifies clinically significant portal hypertension: a prospective multicentre study. Liver Int. 2017; 37: 396-405.

20 Rössle M. TIPS: 25 years later. J. Hepatol. 2013; 59: 1081-93.

21 Merli M, Salerno F, Riggio O et al. Transjugular intrahepatic portosystemic shunt versus endoscopic sclerotheraphy for the prevention of variceal bleeding in cirrhosis: a randomized multicenter trial. Hepatology 1998; 27: 40-45.

22 Salerno F, Merli M, Riggio O et al. Randomized controlled study of TIPS versus paracentesis plus albumin in cirrhosis with severe ascites. Hepatology 2004; 40: 629-35.

23 Sampietro G, Rossi P, Di Marco P. Use of a laryngeal mask in transjugular intrahepatic portosystemic shunt procedures. J. Vasc. Interv. Radiol 1998; 9: 169.

24 Angeloni S, Merli M, Salvatori FM et al. Polytetrafluorethylenecovered stent-graft for TIPS procedure: 1-year patency and clinical results. Am. J. Gastroenterol. 2004; 99: 280-5.

25 Rossi P, Salvatori F, Fanelli F et al. Polytetrafluorethylene-covered nitinol stent-graft for transjugular intrahepatic portosystemic shunt creation: 3-year experience. Radiology 2004; 231: 820-30.

26 Pugh RNH, Murray Lyon IM, Dawson JC. Transection of the oesophagus for bleeding oesophageal varices. Br. J. Surg. 1973; 60: 646-64.

27 Malinchoc M, Kamath PS, Gordon FD, Peine CJ, Rank and ter Borg PC. A model to predict poor survival in patients undergoing transjugular intrahepatic portosystemic shunts. Hepatology 2000; 31: 864-71.

28 Rössle M, Siegerstetter V, Olschewski M, Ochs A, Berger E, Haag K. How much reduction in portal pressure is necessary to prevent variceal rebleeding? A longitudinal study in 225 patients with transjugular intrahepatic portosystemic shunts. Am. J. Gastroenterol. 2001; 96: 3379-83.

29 Silva-Junior G, Turon F, Baiges A et al. Timing affects measurement of portal pressure gradient after placement of transjugular intrahepatic porto-systemic shunts in patients with portal hypertension. Gastroenterology 2017; 152: 1358-65.

30 Karlas T, Lindner F, Tröltzsch M, Keim V. Assessment of spleen stiffness using acoustic radiation force impulse imaging (ARFI): definition of examination standards and impact of breathing maneuvers. Ultraschall Med. 2014; 35: 38-43.

31 Gao J, Ran HT, Ye XP, Zheng YY, Zhang DZ, Wang ZG. The stiffness of the liver and spleen on ARFI Imaging pre and post TIPS placement: a preliminary observation. Clin. Imaging. 2012; 36: 135-41.

32 Ran HT, Ye XP, Zheng YY et al. Spleen stiffness and splenoportal venous flow: assessment before and after transjugular intrahepatic portosystemic shunt placement. J. Ultrasound Med. 2013; 32: 221-8.

33 Novelli PM, Cho K, Rubin JM. Sonographic assessment of spleen stiffness before and after transjugular intrahepatic portosystemic shunt placement with or without concurrent embolization of portal systemic collateral veins in patients with cirrhosis and portal hypertension: a feasibility study. J. Ultrasound Med. 2015; 34: 443-9.

34 Fraquelli M, Giunta M, Pozzi R et al. Feasibility and reproducibility of spleen transient elastography and its role in combination with liver transient elastography for predicting the severity of chronic viral hepatitis. J. Viral Hepat. 2014; 21: 90-98.

35 Gao J, Zheng X, Zheng YY et al. Shear wave elastography of the spleen for monitoring transjugular intrahepatic portosystemic shunt function: a pilot study. J. Ultrasound Med. 2016; 35: 951-8.

36 Singh S, Eaton JE, Murad MH, Tanaka H, Iijima H, Talwalkar JA. Accuracy of spleen stiffness measurement in detection of esophageal varices in patients with chronic liver disease: systematic review and meta-analysis. Clin. Gastroenterol. Hepato. 2014; 12: 935-45.

37 Stefanescu H, Procopet B, Platon-Lupsor M, Bureau C. Is there any place for spleen stiffness measurement in portal hypertension? Am J Gastroenterol 2013; 108: 1660-1.

38 Groszmann RJ, Garcia-Tsao G, Bosch J et al. Beta-blockers to prevent gastroesophageal varices in patients with cirrhosis. N. Engl. J. Med. 2005; 353: 2254-61.

39 Ripoll C, Groszmann RJ, Garcia-Tsao G et al. Hepatic venous pressure gradient predicts clinical decompensation in patients with compensated cirrhosis. Gastroenterology 2007; 133: 481-8.

40 D'Amico G, Garcia-Tsao G, Pagliaro L. Natural history and prognostic indicators of survival in cirrhosis: a systematic review of 118 studies. J. Hepatol. 2006; 44: 217-31. 